\title{
Fetal fibronectin detection for preterm birth prediction
}

\author{
L. Gao ${ }^{1 *}$, J.P. Zhang ${ }^{2 *}$, H. Chen ${ }^{2 *}$, Z.J. Guo ${ }^{2}$, L.B. Chen ${ }^{2}$, J.P. Tan ${ }^{2}$, \\ Y.H. Wang', R. Zhang ${ }^{2}$, Y.L. $\mathrm{Liu}^{2}$ and W.B. Cai ${ }^{3}$ \\ ${ }^{1}$ Department of Clinical Laboratory, \\ The First Affiliated Hospital of Sun Yat-Sen University, Guangzhou, China \\ ${ }^{2}$ Department of Obstetrics and Gynecology, \\ Sun Yat-Sen Memorial Hospital of Sun Yat-Sen University, Guangzhou, China \\ ${ }^{3}$ Department of Biochemistry, Zhongshan Medical School, \\ Sun Yat-Sen University, Guangzhou, China \\ *These authors contributed equally to this study. \\ Corresponding author: W.B. Cai \\ E-mail: weibincai@163.com
}

Genet. Mol. Res. 13 (1): 1323-1328 (2014)

Received January 2, 2013

Accepted July 1, 2013

Published February 28, 2014

DOI http://dx.doi.org/10.4238/2014.February.28.4

ABSTRACT. To study preterm birth prediction based on fetal fibronectin
(fFN) in pregnant women, we randomly selected 124 patients. Vaginal
posterior fornix secretions were analyzed using fFN quick test strips.
Leucorrhea routine samples were collected to detect bacterial vaginosis,
mycoplasma, and chlamydia. Delivery data at 7 days, 14 days, 34 weeks,
and 37 weeks were documented and the sensitivity, specificity, positive
predictive value, and negative predictive value were analyzed. Of the 124
cases, we found $2,4,10$, and 18 cases of maternity within 7 days, 14
days, 34 weeks, and 37 weeks, respectively. The sensitivity, specificity,
positive predictive value, and negative predictive value were as follows:
100, $77.8,6.9$, and $100 \%$ for 7 days; 75 , 78.3 , 10.3 , and $98.9 \%$ for 14
days; $50.0,78.9,17.2$, and $94.7 \%$ for 34 weeks; $33.3,78.3,20.7$, and
$87.4 \%$ for 37 weeks, respectively. Except for 18 preterm births, 23 cases
were fFN-positive, 17 cases had lower genital tract infection. Eighty- 
three cases were fFN-negative, of which 18 cases had the lower genital tract infections. This difference was statistically significant $(\mathrm{P}<0.05)$. Eighteen cases (14.5\% of the pregnant women) had preterm birth. Ten cases delivered within 34 weeks. The negative predictive value and recent predictive value of fFN testing were higher; the positive predictive value was limited due to the impact of lower genital tract infection. The fFNpositive patients need timely clinical processing. During the pregnancy, monitoring of fFN changes and early detection of abnormalities help to reduce perinatal morbidity and mortality.

Key words: Premature prediction; Fetal fibronectin; Negative predictive value

\section{INTRODUCTION}

Preterm birth is an important reason for perinatal death, with an overall incidence rate of $5-15 \%$; the incidence rate is $6-7 \%$ in China. The survival rate of the premature babies was significantly lower than that of full-term babies. Premature babies have a high incidence of some short-term illnesses such as neonatal respiratory distress syndrome, sepsis, pneumonia, intraventricular hemorrhage, and necrotizing enterocolitis. Long-term complications include developmental disorders of the nervous system, cerebral palsy, seizures, vision and hearing impairment, and non-nervous system disorders. The etiology of preterm labor includes numerous factors, but the mechanisms of preterm labor are unclear. Therefore, it is challenging to prevent or treat preterm labor. Currently, many methods can be used to detect preterm birth; fetal fibronectin (fFN) testing of secretions in the cervical posterior fornix is part of evidencebased medicine and is of clear clinical value.

\section{MATERIAL AND METHODS}

\section{Clinical data}

One hundred and twenty-four cases of the outpatients and hospitalized patients were randomly selected for a pregnant check in Sun Yat-Sen Memorial Hospital. These patients were selected from Guangzhou residents from February 2008 to January 2009. The age of the patients ranged from 20 to 42 years. The inclusion criteria were as follows: 1) gestational time of 20 to 34 weeks; 2) no vaginal bleeding, no sexual intercourse within $24 \mathrm{~h}$, no history of vaginal examination; 3 ) cervix dilatation $\leq 2 \mathrm{~cm}$, intact membranes; 4) no other serious pregnancy complications and concurrent disorders such as hypertensive disorders, placenta previa, heart disease, chronic nephritis, viral hepatitis; and 5) single fetus pregnancy.

\section{Research methods}

The rapid fFN test strip (Adeza Biomedical Corporation, Sunnyvale, CA, USA) was used for detection of fFN according to manufacturer instructions. Leucorrhea routine samples were collected to detect bacterial vaginosis, mycoplasma, and chlamydia. 


\section{Statistical methods}

The SPSS 16.0 software package was used for statistical analysis. The rate comparison adopted the $\chi^{2}$ test and non-parametric $\chi^{2}$ test. $\mathrm{P}<0.05$ was considered to be statistically significant. The fFN-predicting preterm statistical indicators were calculated as follows: 1) sensitivity = true-positive patients/total number of patients; 2 ) specificity = true-negative patients/total number of healthy patients; 3 ) positive predictive value $=$ true-positive patients/total number of positive patients; 4) negative predictive value $=$ true-negative patients/total number of negative patients.

\section{RESULTS}

One hundred and twenty-four patients were divided into a symptomatic group and an asymptomatic group. The average age of the patients did not differ between the 2 groups, but the average gestational age differed; thus this grouping was abandoned, and statistical analysis was applied over the entire data set.

\section{fFN testing and delivery time}

The fFN detection was performed on 124 advanced pregnant women, with a gestational time from 20 to 34 weeks. There were $2,4,10$, and 18 cases of maternity within 7 days, 14 days, 34 weeks, and 37 weeks, respectively. The delivery rates showed a statistically significant difference $\left(\chi^{2}=21.462, \mathrm{P}<0.05\right.$; Table 1$)$. The fFN-positive and fFN-negative differences for predicting preterm labor also showed a statistically significant difference $(\mathrm{P}<0.05)$.

\begin{tabular}{|c|c|c|c|c|c|c|c|c|c|}
\hline \multirow[t]{2}{*}{ fFN results } & \multirow[t]{2}{*}{ Cases } & \multicolumn{2}{|c|}{7 days of delivery } & \multicolumn{2}{|c|}{14 days of delivery } & \multicolumn{2}{|c|}{34 weeks of delivery } & \multicolumn{2}{|c|}{37 weeks of delivery } \\
\hline & & $\mathrm{N}$ & $\%$ & $\mathrm{~N}$ & $\%$ & $\mathrm{~N}$ & $\%$ & $\mathrm{~N}$ & $\%$ \\
\hline fFN-positive & 29 & 2 & 6.8 & 3 & 10.3 & 5 & 17.2 & 6 & 20.7 \\
\hline fFN-negative & 95 & 0 & 0 & 1 & 1.1 & 5 & 5.3 & 12 & 12.6 \\
\hline
\end{tabular}

\section{fFN testing value for predicting preterm sensitivity, specificity, positive predictive value, and negative predictive value}

For subjects with 7 days, 14 days, 34 weeks, and 37 weeks of delivery, the sensitivity, specificity, positive predictive value, and negative predictive value, were as follows: 100, 77.8, 6.9 , and $100 \%$ for 7 days; $75,78.3,10.3$, and $98.9 \%$ for 14 days, $50.0,78.9,17.2$, and $94.7 \%$ for 34 weeks; 33.3, 78.3, 20.7, and $87.4 \%$ for 37 weeks, respectively (Table 2).

Table 2. Predicting accuracy of fetal fibronectin in preterm birth.

\begin{tabular}{lcccc}
\hline Delivery time & Sensitivity (\%) & Specificity (\%) & Positive predictive value (\%) & Negative predictive value (\%) \\
\hline 7 days & 100.0 & 77.8 & 6.9 & 100.0 \\
14 days & 5.0 & 78.3 & 10.3 & 98.9 \\
34 weeks & 50.0 & 78.9 & 17.2 & 94.7 \\
37 weeks & 33.3 & 78.3 & 20.7 & 87.4 \\
\hline
\end{tabular}




\section{fFN detection and lower genital tract infection}

Of the 124 cases, except for 18 preterm births, 23 cases were fFN-positive, and 17 cases had lower genital tract infection, accounting for $73.9 \%$ of the pregnant women. Eightythree cases were fFN-negative, of which 18 cases had lower genital tract infections, accounting for $21.7 \%$. This difference was statistically significant $\left(\chi^{2}=22.211, \mathrm{P}<0.05\right.$; Table 3$)$.

\begin{tabular}{|c|c|c|c|}
\hline \multirow[t]{2}{*}{ Group } & \multirow[t]{2}{*}{ Cases } & \multicolumn{2}{|c|}{ Number of the lower genital tract infection } \\
\hline & & Negative & Positive \\
\hline fFN-negative & 83 & 65 & 18 \\
\hline fFN-positive & 23 & 6 & 17 \\
\hline Total & 106 & 71 & 35 \\
\hline
\end{tabular}

\section{Clinical treatment and pregnancy outcome}

During the fFN detection process in 124 subjects, the fFN-positive patients, and the patients with symptoms of preterm labor, were administered tocolytic medication and fetal lung maturity treatment, with successful outcome. Eighteen cases had preterm birth, accounting for $14.5 \%$ of the pregnant women. Ten cases had deliveries within 34 weeks, accounting for $8 \%$. In addition to 2 cases with gestational times $<28$ weeks, all other newborns survived.

\section{DISCUSSION}

\section{fFN levels during pregnancy}

fFN is prevalent in the blood circulation and the amniotic fluid of pregnant women. fFN is generated by extracellular matrix composites between decidua and villi. fFN in the vaginal secretions of 20 -week pregnant women is rare $(<50 \mu \mathrm{g} / \mathrm{L})$ but is elevated at full term (Lockwood et al., 1991). If the fFN level in the cervicovaginal secretions of 20- to 37-week pregnant women is $\geq 50 \mu \mathrm{g} / \mathrm{L}$, the likelihood of preterm delivery is high (Ahner et al., 1995). Some studies (Ahner et al., 1995; Ascarelli and Morrison, 1997) showed that the chorionic and decidual fusion is not sufficiently close during normal early pregnancy, and fFN would leak with cervicovaginal secretions before 20 weeks of gestation. The chorionic and decidual fusion is completed after 20 weeks of pregnancy, and the fFN is then rarely detected in cervicovaginal secretions. When the villi and decidua separation occurs in the lower uterine segments, fFN will release; meanwhile, local inflammation also causes fFN release. At full term, contractions may cause sliding between the fetal membrane and basement membrane and thus restructuring of the cervical collagen organization, resulting in the re-emergence of fFN.

\section{Relationship between fFN and preterm birth}

Contradicting observations have been found regarding the role of fFN for predicting preterm births (Faron et al., 1997; Revah et al., 1998; Moore, 1999; Sakai et al., 2003). Our 
results indicated that fFN had a higher recent predictive value and negative predictive value for predicting preterm birth, but the positive predictive value appeared lower. The proportion of reproductive tract infections of the 124 selected patients was large, and our studies showed that patients with lower rates of genital tract infection had fFN-positive results, whether clinical symptoms were manifested or not. However, the most common causes of premature birth are lower genital tract infection and urinary tract infection, but not all of lower genital tractinfected patients have premature birth. Andrews et al. (2006) found that chlamydia infection during pregnancy does not increase the incidence of preterm birth. We propose that reproductive tract infections interfere with $\mathrm{fFN}$ testing during pregnancy, influencing the positive predictive value; therefore, fFN detection during the middle or late phases of pregnancy may be significantly improved by excluding patients with lower genital tract infection. Unfortunately, the reproductive tract infection rate was high in our data set, accounting for $45.2 \%$ of the pregnant women in this study, and the sample size was too small after excluding the lower genital tract infection. The fFN-positive samples comprised 9 cases of which 3 had the premature births. The fFN-negative samples corresponded to 72 cases, of which 7 cases had preterm births. Therefore, the statistical analysis was not performed after the exclusion.

\section{fFN and preterm birth prevention}

The value of using fFN as an indicator for predicting preterm births is widely recognized, and some researchers (Lee et al., 2009) have suggested that fFN detection can be used as a first-line predictor for premature birth in pregnant women with a history of preterm birth. In the present study, we found that the negative predictive value and the recent predictive value of fFN testing were higher, but the positive predictive value was limited due to the impact of lower genital tract infection (Sanchez-Ramos et al., 2009). If subjects with reproductive tract infections are not subjected to the test, the positive predictive value of the application will be greatly enhanced. According to the characteristics of the high negative predictive value and the recent predictive value in clinical practices, fFN-negative patients do not need to be over-processed, saving medical resources. For fFN-positive patients, other premature birth prediction methods, such as the B ultrasound measurement of the cervical length, can be used for accurate diagnosis (Bolt et al., 2011). Prediction of preterm births should be a continuous process, and patients with risk factors should be subjected to several detection methods to determine the likelihood of preterm birth. Repeat testing is needed for early detection, diagnosis, and treatment.

\section{ACKNOWLEDGMENTS}

Research supported partially by the National Nature Science Foundation of China (\#81170625, \#81000259, \#30973207, and \#81070746), the Guangdong Natural Science Foundation (\#10151008901000007 and \#10451008901004246), the Fundamental Research Funds for the Central Universities (\#3161001), the Fundamental Research Funds for the Central Universities (Youth Program \#09YKPY73, \#12ykpy29), and the Research Fund for the Doctoral Program of Higher Education of China (\#20090171120075).

\section{REFERENCES}

Ahner R, Kiss H, Egarter C, Zeillinger R, et al. (1995). Fetal fibronectin as a marker to predict the onset of term labor and delivery. Am. J. Obstet. Gynecol. 172: 134-137. 
Andrews WW, Klebanoff MA, Thom EA, Hauth JC, et al. (2006). Midpregnancy genitourinary tract infection with Chlamydia trachomatis: association with subsequent preterm delivery in women with bacterial vaginosis and Trichomonas vaginalis. Am. J. Obstet. Gynecol. 194: 493-500.

Ascarelli MH and Morrison JC (1997). Use of fetal fibronectin in clinical practice. Obstet. Gynecol. Surv. 52: S1-12.

Bolt LA, Chandiramani M, De Greeff A, Seed PT, et al. (2011). The value of combined cervical length measurement and fetal fibronectin testing to predict spontaneous preterm birth in asymptomatic high-risk women. J. Matern. Fetal Neonatal Med. 24: 928-932.

Faron G, Boulvain M, Lescrainier JP and Vokaer A (1997). A single cervical fetal fibronectin screening test in a population at low risk for preterm delivery: an improvement on clinical indicators? Br. J. Obstet. Gynaecol. 104: 697-701.

Lee HJ, Park TC and Norwitz ER (2009). Management of pregnancies with cervical shortening: a very short cervix is a very big problem. Rev. Obstet. Gynecol. 2: 107-115.

Lockwood CJ, Senyei AE, Dische MR, Casal D, et al. (1991). Fetal fibronectin in cervical and vaginal secretions as a predictor of preterm delivery. N. Engl. J. Med. 325: 669-674.

Moore ML (1999). Biochemical markers for preterm labor and birth: what is their role in the care of pregnant women? MCN Am. J. Matern. Child Nurs. 24: 80-86.

Revah A, Hannah ME and Sue AQA (1998). Fetal fibronectin as a predictor of preterm birth: an overview. Am. J. Perinatol. 15: 613-621.

Sakai M, Sasaki Y, Yamagishi N, Tanebe K, et al. (2003). The preterm labor index and fetal fibronectin for prediction of preterm delivery with intact membranes. Obstet. Gynecol. 101: 123-128.

Sanchez-Ramos L, Delke I, Zamora J and Kaunitz AM (2009). Fetal fibronectin as a short-term predictor of preterm birth in symptomatic patients: a meta-analysis. Obstet. Gynecol. 114: 631-640. 